\title{
Big Governance Research: Institutional Constraints, the Validity Gap and BIM
}

\author{
Peter P. Houtzager and Arnab Acharya
}

\begin{abstract}
1 Introduction
The pressing questions about governance today require research on a scale, and of a complexity, that the existing institutional environment for research has great difficulty supporting. This article identifies some of the current institutional constraints on governance research, and examines a set of institutional innovations that enable a form of 'big governance research' that begins to meet the information and knowledge requirements of contemporary governance questions. It presents the organisation and methodology of the multi-country study 'Modes of Service Delivery, Collective Action and Social Accountability in Brazil, India, and Mexico' (henceforth BIM, for Brazil, India and Mexico). We argue that the organisational and funding model that BIM has created permits the type of interdisciplinary, process-oriented, and multi-country or multi-region research needed to answer governance questions of international concern.
\end{abstract}

Governance research takes many forms but its central ambition is to identify the complex processes of change that contribute to better governance, and to draw broad lessons about these processes that will hold for a range of countries or contexts. The methodological requirements for conducting such research are not only non-trivial, but require an institutional research environment that is substantially different from the one that prevails today.

The research designs that are particularly well suited to address governance questions are interdisciplinary, process-oriented and multi-country or multi-region comparisons. Such research is logistically complex, requires relatively high levels of funding, and by social science standards, long time frames. The structure of national universities and research institutes, as well as that of many international organisations, favours disciplinary research, conducted within relatively short time frames, and using either quantitative snap-shots (even in the case of panel data) or a small number of case studies.

The mismatch between complex questions that cover a diverse range of contexts and the methodological choices available in the current research environment leads to a substantial 'validity gap'. That gap is between the type of information required to answer our questions and the type our methods can produce, and is often so large that few if any generalisations can be reasonably be supported by the research. The institutional constraints on methods are so severe that there is a powerful argument for new institutional designs to support international governance research.

The BIM study makes progress towards establishing an alternative mode of organising, financing and conducting multi-country research. The study is located in the Centre for the Future State (CFS), a UK government-funded development research centre based at the Institute of Development Studies (IDS). The CFS brings together research networks in eight countries through its partner institutions. These networks, and the ability of the CFS to make medium-term investments, enables interdisciplinary multi-country studies through well-coupled interdisciplinary research teams that conduct rigorously comparative research - i.e. working within a coherent analytic framework, using a single comparative methodology, and conducting fieldwork in comparable ways in disparate research sites. BIM itself is a collaboration between researchers located in the UK, São Paulo, Delhi and Mexico City. The study explores how recent reforms of the institutions 
that provide public services shape the capacity of organisations representing the poor to negotiate public policy and engage in social accountability of providers.

The next section provides a brief summary of what we mean by validity and the possible sources of validity gaps. We then explore some of the institutional constraints on the research methodology, and two common models for organising multi-country studies that suffer distinct validity gaps. Turning to BIM, we first discuss the institutional environment in which the study was developed and then the methodology itself.

\section{Three components of a research methodology}

To draw valid conclusions or make valid generalisations, our methods must produce the information required to answer, or at least explore, our questions. Slippage between these knowledge requirements and that which our methods produce can occur between the three basic and interrelated components of virtually any research design: conceptual, measurement (or data-gathering) and causal analysis. Each component has to have a degree of alignment or congruence with the others.

\subsection{Conceptual validity}

Conceptual validity is how we break down the large concepts in the research question - such as reform of service provision, representation, policy process or social accountability - into constitutive dimensions that can be observed or measured. The definition or operationalisation of a concept should identify its empirical referents as unambiguously and accurately as possible (Adcock and Collier 2001; Gerring 2001: 48-50). Conceptual validity is a particular concern in multi-country studies: political representation in one democratic polity may have only one dimension, representation through elected legislative bodies and/or executive, but in another polity may have additional dimensions, such as through corporatist institutions and/or participatory governance institutions.

\subsection{Measurement validity}

Measurement validity is the accuracy with which we measure 'what we think we are measuring', or the extent to which empirical indicators to measure a concept's dimensions (as defined above) effectively capture those dimensions (King et al. 1994: 25). In multi-country studies, there is a particular concern with the comparability of variables that will be measured; for example the poverty line may be measured differently in different countries, but the use of a line should carry similar meaning across countries.

\subsection{Causal validity}

Causal validity is the strategy for establishing whether or not there is a causal link between a set of factors believed to contribute to an outcome, such as a reduction in corruption, higher school attendance, or decline in ethnic violence. The strategy must be able to identify and rule out competing or alternative explanations, and delimit the range of contexts in which the identified causal link holds. Causal validity is low, for example, when a study attempts to establish a causal link between social accountability and greater access to healthcare, and conducts research only in areas where access to healthcare is high.

Validity is how accurately (or defensibly) each of these components captures or corresponds to the other. It is possible that there is a small analytic gap between the research question and the analytic method, but due to poor operationalisation there is a large gap between the dimensions of the study's central concepts and the ways these are measured empirically. The researcher's challenge is to ensure that the cumulative validity gap is not so large as to invalidate generalisations or conclusions drawn from the research.

\section{International research in a parochial (or not very globalised) world}

Researchers in the physical or social sciences often work in a context of pervasive constraints, and the optimal research design is out of reach. Many of these constraints are institutional, though certainly not all. Given these constraints, matching questions and methods carefully is vital to reducing the validity gap.

Governance research attempts to identify factors or processes which contribute to (mostly positive) forms of change, across a wide array of contexts. It is foremost concerned with change - i.e. in processes that over time contribute to desired change (such as greater accountability) that block desired change (corruption in service delivery), or that lead to undesired change (civil war, regression to authoritarianism). Having identified that positive, negative, or no change has occurred, governance research is expected to identify why or what combination of factors contribute to the uptake of new policing technologies, or to the lack of 
compliance with seat-belt laws. The knowledge requirements to address these questions are often cross-disciplinary and go beyond national boundaries. They are, furthermore, not formulated to produce high-level theory, but to produce generalisations applicable to quite different national or sub-national contexts. This reflects the requirements of public policy, which can only apply large-scale solutions, and of international organisations that operate in many different contexts in particular.

In international governance research, when we seek to generalise across countries or regions we often find that if we do not tolerate a large validity gap, the methods we are able to deploy only allow us to answer the simplest and least interesting questions. ${ }^{1}$ We face validity gaps because the governance questions are ambitious and complex while the institutional context, which supports research, is overwhelmingly parochial in both disciplinary and national terms, and often has short time frames. This should not be entirely surprising, given how closely the emergence of the major research centres is tied to the emergence and consolidation of the nationstate. Our research environment was largely designed for national or sub-national research, or research in the national interest, narrowly defined. Comparative social science research on any scale is very young and closely tied to the national interests of the different states by which it has been sponsored. Research institutions therefore tend to foster disciplinary, short-term and/or national collaborations.

The institutional constraints on methodological options can be of three broad types. First, there are powerful disincentives to interdisciplinary research in most institutional contexts. Leaving aside imponderables such as 'what counts as knowledge', disciplines have their own (1) specialised languages that involve substantial costs to translate or assimilate, (2) professional incentives to engage with particular issues and/or adopt particular methodologies, and (3) publications, conferences, and so forth that direct the timing and ways in which researchers work.

Second, researchers located in different countries face additional and distinct institutional barriers to international collaboration beyond ordinary language barriers. Disciplines in different countries have their own national trajectories, emphasising particular research areas, methodologies, and forms of exposition. As a consequence, it remains surprisingly difficult, for example, for leading Latin American scholars to publish in English language journals, even within their own discipline. The types of institutional and professional obligations researchers have also vary substantially across countries, imposing quite distinct time demands. Because of these national differences, there are particularly strong incentives for researchers to favour work rewarded within their national context rather than internationally, even if the latter brings a degree of local prestige. One of the biggest challenges to constituting a multi-national research team, one in which researchers remain in their home institutions, is countering powerful local incentives with some form of overarching 'regime' with its own 'rewards and punishments'.

One important difference between researchers, which cuts across disciplinary and national boundaries, is whether they are located in hard or soft money institutions. Soft money institutes like the Institute of Development Studies (IDS) in the UK and Centro Brasileiro de Analise e Pesquisa (Brazilian Centre for Analysis and Planning, CEBRAP) in São Paulo need to secure funding year-to-year and generally suffer from short funding cycles. On the one hand, the continual search for research grants, consultancies and so forth, puts a premium on raising funds, pushing researchers towards undertaking successive short research projects and underexploring the information these projects collect. On the other hand, soft money institutes are particularly open to collaboration and to interdisciplinary research as they appear to produce an entrepreneurialism and willingness to experiment. They also tend to develop accounting systems, organisational structures, and so forth that facilitate collaboration between different types of institutions.

Hard money institutions however, such as departments in public universities, are particularly closed to collaboration, in part because they already have assured institutional funding and in part because they are part of large complex bureaucracies. Not only is moving resources between hard money collaborators difficult (and in some cases departments have little control over whether the resources arrive), but university departments can, on the one hand, encourage research with longer time frames and allow faculty to exploit their data more fully. On the other hand, there are few incentives to engage with what is often an uncertain and 
complicated process of international collaboration. Their salaries and professional status are largely independent of such collaboration.

Third, the funding structure for governance research strongly favours short-term projects. Paradoxically, more 'international' sources of funding, such as the US foundations, bilateral donors, or the World Health Organization (WHO), Food and Agriculture Organization (FAO) or World Bank, generally favour shorter-term horizons for the research they fund than national sources, albeit each for their own reasons. More 'national' funding sources, such as the Economic and Social Research Council (ESRC) in the UK, Fundação de Amparo à Pesquisa do Estado de São Paulo (FAPESP) in Brazil, or the National Science Foundation in the USA, tend to have longer time frames and are more likely to sponsor the creation of specialised research networks or centres, but within their respective national boundaries.

\subsection{Two responses to institutional constraints}

If the institutional environment inhibits more interdisciplinary, process-oriented, and multi-country research, requiring medium- to long-term time frames and higher funding levels, then what types of research is most common today and how is it organised? To our knowledge, there is no data on the different forms international governance research has taken, but experience suggests that two general models are common. These models apply as well to within-country studies that seek variation either across regions of the country or over distinct periods of time. ${ }^{2}$

One model is the loosely coupled research network, which can be either interdisciplinary or disciplinary. These networks are constituted by groups of researchers, located in their respective institutions, who have horizontal ties that facilitate research around a particular theme or set of issues, while leaving each to define their own methods and/or case selection. The strength of loose-coupling is coverage, many research teams from diverse national contexts can be incorporated, and the production of exploratory work in areas where little information is available. ${ }^{3}$ The weakness of such networks is the high levels of variation in methods, and sometimes even theoretical framework. In interdisciplinary networks, such as that constituted by the Ford Foundation, this variation matches the high level of coverage achieved.
Research conducted within loosely coupled and interdisciplinary networks is particularly vulnerable to comparative anecdotalism, i.e. making generalisations from case studies that are not comparable, either because they are of different phenomena or because they are situated in dissimilar contexts. Many of the initial case studies of citizens' participation (or direct democracy) in low- and middle-income countries, for example, are detailed examinations of particular civil society actor organisations or of specific types of participatory institutions or experiences. ${ }^{4}$ These freestanding case studies have provided a wealth of information and insights. It is in the effort to draw broad lessons or make generalisations from these case studies that authors have engaged in forms of comparative anecdotalism - i.e. idiosyncratic cases from different contexts are herded together into a single explanation. These cases, however, are rarely comparable. They are either not instances of the same things, or they occur in dissimilar sociopolitical contexts (Gurza Lavalle et al. 2005).

The second model is a multi-context hierarchy. $A$ single scholar or institution coordinates a small research team that conducts research in a number of countries. Often local researchers or institutes are contracted to execute particular pieces of research, but there is no effective collaboration. International organisations, such as the FAO, WHO or the International Development Research Centre (IDRC), tend to conduct, or commission, research within such multi-context hierarchies.

A particular form of such hierarchies is quantitative research conducted by a single team, located in one institution, using large datasets that include a number of countries. Quantification is one solution to the institutional constraints international research faces - the creation of a single metric, summarised in an index, for complex and variable phenomena, such as corruption or political freedom, makes possible comparative statements. The quantification solution is an attractive one and has gained adherence, but it is not without its own particular set of challenges. ${ }^{5}$ Quantification of governance phenomena involves setting a measurement standard, relevant and acceptable to (or imposable on) diverse groups, and a method for generating the quantitative data. Contrary to data on the economy, such as current accounts, for which governments have developed relatively elaborate reporting systems, even the most basic governance data is difficult to come by, such as 
that on judicial procedures, civil society organisations, or legislative action.

In place of a system for data collection, quantitative studies increasingly rely on country 'experts' to score dimensions of governance and then construct indices and country rankings using these scores. The expert judgements, however, are entirely subjective and the approach is not in fact comparative: different people, with their respective subjectivities and knowledge limitations, score the country on which they are considered an expert. Furthermore, in the absence of 'big governance research' that can identify the causal processes that shape contemporary governance, these quantitative studies implicitly or explicitly impose their own notions of what causal processes are real and matter for governance when selecting which dimensions of governance ought to be measured and brought into their indices. Ideas about what forces shape governance are not tested empirically, but imported into the very indices that are later used in quantitative analyses. ${ }^{6}$

\section{BIM: research in a well-coupled interdisciplinary network}

How is BIM's research methodology different from these two models, and what are the characteristics of the institutional foundations that allow a different model of multi-country research? The study is being conducted collaboratively by research teams located in three countries, using the same causal, conceptual, and measurement/data-gathering strategies. The process of constructing this methodology is closely connected to the formation of the multi-country research team, and this connection is highlighted below.

The well-coupled interdisciplinary network created by the CFS, along with IDS and CEBRAP in São Paulo, has made possible a medium-term investment in constructing, and subsequent coordination, of a multi-country research team and a relatively sophisticated methodology. In particular, it allowed for a 1.5-year upfront investment in: choosing research sites to compare; selecting comparable service sectors and specific programmes to compare; constructing a methodology that combines research on processes, structures, and outcomes of the interaction between organisations representing people in poverty, policymakers and providers; regular international meetings among researchers to produce a common sense of the project's framework and methods, i.e. convergence; and systematic professional training of young researchers.

\subsection{Centre for the Future State (CFS)}

The key institutional component that has made BIM possible is the virtual CFS, one of several development research centres funded by the UK Department for International Development (DFID). The CFS is located at IDS and funded in 5-7 year blocks of time, at a substantial level: $£ 2.5$ million. It is composed by eight research institutions located in Brazil, Ghana, India, Indonesia, Kenya, Pakistan, South Africa and the UK. It differs from many other networks, such as academic disciplinary or area studies associations, or those of international organisations.

Programmatic but interdisciplinary orientation. The researchers in the CFS come from a number of disciplines but share a common programmatic interest in 'building more effective, accountable and responsive governance in poor countries'?

Researchers come from various social science disciplines and country specialisations.

Strong institutional incentives fostering collaboration. These incentives include, most obviously, funding for research at substantial levels and international exposure, including that which facilitates South-South comparative research. There are also more subtle incentives, including participation in a high calibre intellectual environment, access to international literatures, opportunity to write up research away from one's home institute, and specialised training in methods such as network analysis. Critically, these incentives have to compete with those of researchers' home institutions, without eroding the researchers' position in those institutions.

Capacity building of a new generation of 'comparative researchers'. One of the major challenges facing longer-term collaborative research and institutionalising research programmes is locating and (especially) retaining talented young researchers. In order to address this challenge in the CFS institutions, the centre has made a significant investment in the professional development of young researchers, with strong incentives for these researchers to commit (over the medium term) to their home institutions. This includes linking participation in research, to obtaining professional degrees, providing specialised training and broad intellectual support. 
Stable relations which allow debate and collaboration to evolve over extended periods of time. Among the more powerful obstacles to international research are the national research and academic traditions, which differ in analytic and methodological approaches, as well as in styles of argumentation and exposition. Leaving aside the difficulties inherent to translating social science concepts into different languages, ongoing interaction around a core set of ideas helps produce some degree of intellectual convergence, or at least sufficient empathy to make true debate possible.

\subsection{Constructing team and method}

These features of the CFS allowed the BIM study to address some of the institutional constraints on establishing interdisciplinary and multi-country research team, including the higher costs and long time frame required. From the initial discussions of the project in mid-2005 to initiating fieldwork in early 2007, the CFS supported a series of international meetings attended by potential participants of the study. Additional meetings took place once a year alongside the CFS annual meetings. At each of these meetings the composition of the team, the choice of research sites, and the methodology were defined more sharply.

The project uses three distinct levels of analysis that captures the processes of bargaining and negotiation between state and society actors, as well as the more quantitative or structural snapshots at several points in time. The study examines changes in the interaction between collective actors (or civil society organisations) who represent poor communities on the one hand, and policymakers and providers on the other, at three distinct points in time: in the period immediately prior to the reforms (our baseline), during negotiation of the reforms themselves, and in the post-reform period.

Cross-national research requires balancing (a) comparability across contexts with sensitivity to local context, and (b) simplicity and formalisation of design to reduce human error with enough nuance and flexibility to capture multi-dimensional and intricate social processes. Establishing comparability requires a high level of prior knowledge of each of the research contexts - in our case, the cities and the reform processes - and substantial conceptual work in defining levels at which comparison is possible and analytically useful or interesting. Simple and formalised research designs, like that used in survey research or network analysis, also require a substantial upfront investment.

The study has invested in developing a common language and 'common sense' of the study's framework, goals, and substantive knowledge of public sector reforms. We are engaged in a resource intensive process that allows for substantial amount of face-to-face time, within city-team discussions, training, and a degree of formalisation of the methods. Young researchers in particular are benefiting from this process. They have an exceptional opportunity to participate in the process of designing research, conducting fieldwork, participation in discussions of literature, and obtain South-South exposure. Furthermore they are strongly encouraged to obtain professional degrees as part of their involvement in the study. Eleven young researchers from Brazil, India, Mexico and the UK have in different ways contributed to the study and appropriated it to pursue their professional goals. Most have had exposure to annual CFS discussions in Brighton and about half have travelled to each other's countries to accompany fieldwork.

\subsection{Choice of research sites: three cities}

The choice of Delhi, Mexico City and São Paulo is based on pragmatic and analytic criteria. The pragmatic criteria include knowledge of the researchers in the CFS network, and the availability and interest of these to engage in the study. The analytic criteria include the focus on large urban centres where reforms of public service delivery have been substantial. The comparative strategy between the cities emerged out of the intersection of these two.

The final three cities in the study are 'critical cases'. That is, they are cases where the study's hypotheses are most likely to hold true. This delimits the range of empirical phenomena to which our findings can be applied with confidence. If the hypotheses do not hold in these contexts, they are less likely to hold elsewhere.

Relative to other regions in the three countries, and to other low- or middle-income countries, Delhi, Mexico City and São Paulo have more active civil societies, have a substantial history of service delivery by public agencies, and are focal points for public policy. It is in the these cities where national reforms can be expected to be made, where collective action 
is likely, and where one would expect changes in the pattern of state-society interactions around the services to emerge. In addition, the choice of large urban centres reflects a concern with having relatively homogenous analytic units in countries with tremendous regional variation - in state presence, density of civil society, and so forth. The cities therefore permit a far more detailed and nuanced causal assessment than national-level studies.

\subsection{Selection of sectors: establishing comparability} The choice of sectors in each country/city was driven entirely by analytic concerns. Two sectors were selected that had undergone significant reform but that differed in the nature of the 'goods' they provide, and in other sector-specific factors, such as differences in the ideology of sector's professionals or the strength of their organisations. This variation across the pairs in each city provides some control for these sector-specific factors.

There is surprisingly little published material comparing the second wave of public sector reforms across countries, and none that makes comparisons at the municipal level. Establishing comparability between reform processes in each country and city therefore required developing substantial knowledge of each and undertaking conceptual work to define levels at which comparison is possible and analytically productive.

The first step in selecting sectors and programmes was to survey the secondary literature and conduct initial interviews with experts, to produce overviews of national public sector reforms since 1985 in seven sectors: health, education, social assistance, electricity, sanitation (water and sewerage), public housing and public transport. These overviews provide a panoramic view of recent voice- and choice-oriented reforms, or absence thereof in some cases, across the countries and metropolitan areas: they summarise the types of national-level reforms (decentralisation, pluralisation of providers, participation of users) and whether the sector was one in which networks of civil society actors participated in the policymaking process or pressured government from the outside.

Healthcare and social assistance were selected because they are directly related to poverty reduction, have seen significant institutional reforms in the mid1980 s to the early 2000s, and represent distinct types of public goods. In addition, collective actors had been prominent in the design or implementation of some of the reforms in the sectors, but not in others, providing additional variation.

Research was then conducted for narratives of national-level reforms in each sector. These papers became the training ground for the young researchers in writing analytic papers. The papers became the bases for articles that seek to offer a new interpretation of the reforms to the national literature on the topic, providing the researchers their first publications.

Summarising briefly, in Brazil health reforms have been more voice-oriented - universalisation of access to healthcare has been accompanied by significant decentralisation and the creation of deliberative policy councils, in which collective actors representing diverse interests have legally guaranteed seats. Within social assistance, there are several distinct areas and the study focuses on poverty reduction, which has moved towards a choice model that in some cases is rights-based (universal minimum income guarantees) and in others, closer to conditional cash transfers (CCTs), with narrow targeting. In Mexico health sector reforms and poverty reduction have been, broadly speaking, choice-oriented. In healthcare successive reforms have focused on decentralisation and pluralisation of providers, particularly by supporting the development of the healthcare market. Similar to Brazil, however, the national CCT (Oportunidades) is the most significant form of poverty reduction initiative (see Hevia, this IDS Bulletin). The reforms in Delhi differ in nature, in that they are broader institutional reforms of the state rather than sectoral ones. These broader reforms, such as decentralisation (through the 74th constitutional amendment) and the Right to Information Act, share a part of the voice logic of the Latin America reforms, as they seek to enhance citizens' rights and leverage vis-à-vis public bureaucracies.

In each sector, a specific programme was selected that is executed by the municipal government and that embodies the institutional logic of the larger reforms. Within health, for example, we have selected Reproductive Health in Delhi and Mexico City, and the Family Health Programme in São Paulo. Participation of collective actors (representing women) in the design of these programmes appears to be common, even in Mexico. In India, the logic of the health sector at the national level has been 


\section{Figure 1 Project multi-level analysis}

The diagram illustrates an increase in capacity of civil society actors, as the number of actors and their connectedness and ties to public officials grow. $T_{1}$ represents the period prior to reforms and $T_{2}$ the period after.

\section{Analytic levels}

1. Narrative account of national-level politics of reforms and municipal-level case studies of designing/implementing programmes that embody logic of the reforms
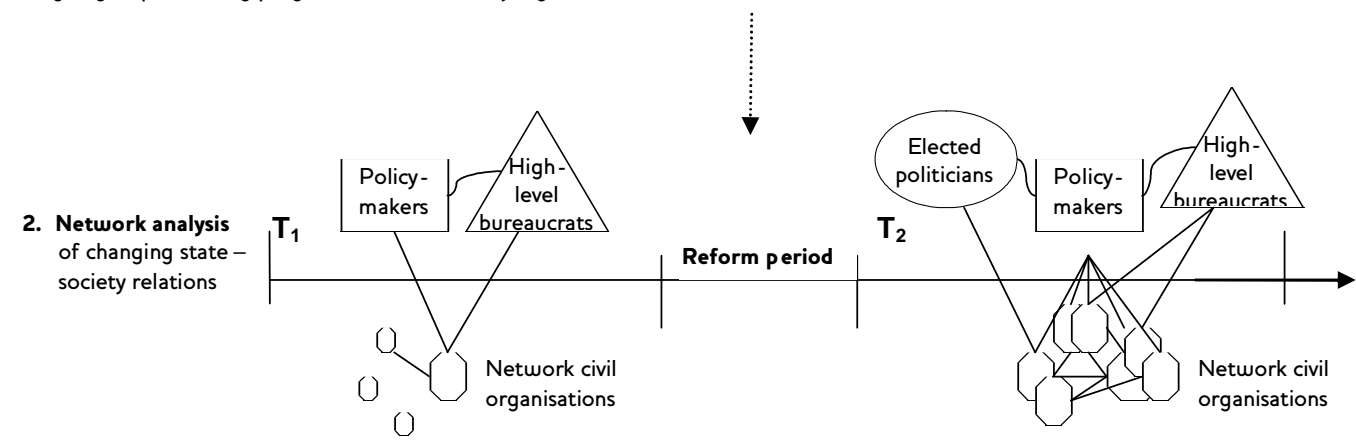

(

3. Cross -sectional analysis of 40 catchment areas in low income areas that vary in levels o $\mathrm{f}$ collective action to identify any impact on (i) policy implementation and ( ii) service outputs

historically driven by international donors and multilateral agencies' population control agenda and, as the broader field of reproductive health has become well established, it has also became a target for collective action. In Brazil the federally funded but municipally executed Family Health Programme has sought to operationalise the aspirations and policies of the national reforms creating the Unified Health System (see Dowbor, this IDS Bulletin). Its design reflects the major participatory trends in the health sector since 1988. In the case of social assistance, we selected a minimum income guarantee programme in São Paulo and universal pension programme in Mexico City. These are choice reform programmes advocated by international actors and which will have little participation or social accountability. In India the anti-poverty programme for the food security Public Distribution System (PDS) and its shift to the Targeted Public Distribution System was selected.

\section{Causal (multi-level) strategy}

The causal strategy consists of three levels of analysis that seek to meet the process-oriented nature of our question and the production of rigorously comparable data in each of the cities. The three levels are: (1) movie-like case studies that trace processes of change over time, narrating the negotiations of policy reforms at the national level and of specific programmes at the municipal level; (2) snapshots at two points in time of the structure of the network of actors representing the poor and of their relations to policymakers, that portray in quantitative ways the changes in access to policy negotiations (and leverage in these) before and after the reforms; and (3) quantitative snapshots of different regions of the city, at a single point in time, to assess whether social accountability is more likely where local organisations are connected to actors who negotiate policy, and whether social accountability in general contributes to greater uptake of reforms and improved services.

The design and operationalisation of this multi-level research strategy has required over a year of intensive interaction among team members distributed across the three sites and the UK. This long lead-up period reflects in part the need to formalise the relatively sophisticated methodology, as 
each component had to be operationalised through relatively simple fieldwork modules that would ensure comparable data is produced in each city. These modules in turn allow for a degree of local adaptation and improvisation.

For each of the levels, we therefore wrote detailed methods notes which were then discussed in reading groups and ultimately in larger international team meetings. This process led to demands from team members for further methods training, particularly in network analysis. Subsequent to the methods notes, we wrote detailed fieldwork notes that broke major concepts into several dimensions, for which it was then possible to identify empirical indicators. These notes explained the move from concepts to measurement, and a strategy for collecting the needed data, and are a particularly important part of the formalisation process.

\subsection{1st analytic level - process tracing through case studies}

In order to trace the processes of change-over-time, detailed case study work is being conducted. The case studies of the policy reform episodes allow us to trace the process of interaction, and in some cases negotiation, between actors representing poor (under-served) communities and policymakers in state departments. This makes it possible to identify the extent to which these actors had a role in negotiating reforms, at national and city levels, and whether they had an opportunity to construct some form of institutional fit to lock-in their long-term engagement with the state (Houtzager 2003; Joshi, this IDS Bulletin). The construction of institutional fit will be visible in the crystallisation of specific formal, and in some cases informal, institutional mechanisms that link collective actors and policymakers.

The cases studies also allow us to assess the responsiveness of public policy to distinct actors, by matching demands of such actors to the content of policies that were adopted. They provide the information necessary to interpret the networks of actors representing the poor and their relations to policymakers and political leaders. Network data, like quantitative data, does not 'speak' for itself depending on one's interpretive framework the data can be read to support quite different arguments or hypotheses. It helps, for example, to establish what it is that flows through the networks that provide its leverage in public policy, or which alliances were particularly important. Or, how a particular reform contributed to densifying or institutionalising a network, or to adding or shedding particular segments.

\subsection{2nd analytic level - network analysis}

One of the study's innovations is to use network analysis to explore in a more systematically comparative way the changing patterns of relations among collective actors who represent the poor and in their relations to policymakers. The formalisation involved facilitates comparisons across time periods and sectors, as well as comparisons between the cities. When combined with detailed qualitative and contextual data, it allows one to identify changes in the structural capacity for action of different collective actors and of their network as a whole (Diani and McAdam 2005).

The well-coupled nature of the BIM research network and the ability to invest CFS time and resources have been particularly important to developing the network analysis component of the study. The opportunity to adopt network analysis came from CEBRAP, where researchers have been using such analyses to explore public policy in São Paulo. Studies of policy networks conducted in affluent Western democracies have focused on formal relations between organisations, rather than on relations between individuals that link those organisations through both formal and informal ties (Knoke et al. 1996). The assumption that formal relations between organisations matter more than relations between individual leaders of organisations may not, however, hold up in Brazil, India and Mexico. We therefore shift the focus from organisations to individual leaders. We are constructing networks using relations between individual leaders of organisations, on whom we then map formal organisations. We thus take into account an important role that informal relations play in policymaking and in the construction of political alliances, as well as trace the movement of key leaders between organisations and into the state (Marques 2000, 2004). To identify changes in the capacity of networks of collective actors to influence policy, we are constructing the networks at two moments in time: prior to reforms and after the reforms (at least five years on from the last major policy changes). The attributes of actors and measures of the network, as well as the positions of prominent actors within those (such as actors that link large numbers of actors to policymakers) provide 
quantitative-structural view of increased or decreased access to policy negotiations (Carrington et al. 2005).

\subsection{3rd analytic level - social accountability of providers}

The inclusion into the research network of an economist with experience in evaluation studies permits the study to explore a cross-section of regions in each city to gauge whether local organisations that target local service providers - as a form of social accountability - has any effect on the uptake of reforms and on access and quality of services. A stratified sample of approximately 40 evaluation units, where we believe uptake and quality of health and social services varies along with the intensity of collective action, is being drawn from low-income regions of each city. Local collective action around service delivery is being measured along a few key dimensions of collective action for which it is possible to establish indicators, such the networked-ness of collective actors and the degree of institutionalisation of their demand-making and monitoring of services. The evaluation of uptake of reforms and service delivery performance will be assessed through measures of policy implementation, service output, and where data availability allows, impact on wellbeing. ${ }^{8}$

\section{Conclusion}

The BIM study, we believe, provides an indication of the types of methodological strategies, and the

\section{Notes}

1 The dimension of this challenge depends in part on the types of questions we pose: overly general or poorly developed questions pose insurmountable obstacles to developing a coherent methodology.

2 Within-country studies have the advantage of controlling the variation of many variables, but cannot address which different national contextual factors affect the phenomena under investigation, nor why this may be the case.

3 There are also loosely coupled disciplinary research networks, in which researchers from different institutions (normally within the same country) agree to address a particular question within a single broad framework. The individuals tend to be country specialists and may employ different methods, but they are engaged in a dialogue within a particular sub-field (cf. the Johns Hopkins University Press series Democratic Transition and Consolidation). required institutional foundations, that reduce the validity gap in international governance research. It shows what is possible even within the limits of a relatively small well-coupled research network, when medium-term funding is available. The findings the study is producing, and in particular the generalisations based on these findings, nonetheless only cover contexts similar to those of the three urban centres. If our hypotheses prove correct in these contexts, further research is needed to assess whether they hold in rural areas, in smaller cities, and so forth. If they are not correct, they can be ruled out for most contexts, because the cities are critical cases.

Notwithstanding these limitations, BIM points to the need to engineer a research environment that is more favourable to multi-country governance research. The social sciences often look towards the physical sciences for metaphors, i.e. causal 'mechanisms', social 'movements', 'networks'. It may be time to look towards 'big science' for the types of institutional structures that can support 'big governance research' - larger in geographic and methodological coverage, and hence in time frame and funding. This is not a call for a social science equivalent of a nuclear particle accelerator, but for new coordination mechanisms that allow for a scale and scope of research that matches that of our governance questions.

$4 \mathrm{Cf}$. the large multi-country projects undertaken by Santos, with MacArthur Foundation support, 'Reinventing Social Emancipation', www.ces.fe.uc.pt/emancipa; the Ford Foundation, 'Civil Society and Governance Project', www.ids.ac.uk/ids/civsoc/index.html and on Brazil specifically, see Dagnino (2003); as well as research from the the Johns Hopkins Center for Civil Society Studies, www.jhu.edu/ccss/

5 Another response has been to construct deductive theories of bad or good governance which elide the problem of empirical validation altogether. Some of the social choice models, relying on different theories of incentives, used for example to defend particular property rights regimes, offer a good illustration

6 See the International Country Risk Guide (ICRG), discussed in Moore et al. (2003), as well as the Corruption Perception Index and Freedom House's 'Freedom in the World Country Ratings'. 
7 The CFS also shares a broad framework, in that it sees 'the interaction between state and society as playing the central role in constructing effective, accountable public institutions. These evolve through a political process of bargaining between the state and organised groups in society. They cannot be generated simply by transferring institutional models from rich to poor countries'. The CFS' most interesting findings from 2001-5 can be found in Changing Paths (2006), www.ids.ac.uk/futurestate/

\section{References}

Adcock, Robert and Collier, David (2001) 'Measurement Validity: A Shared Standard for Qualitative and Quantitative Research', American Political Science Review 95.3: 529-46

Carrington, Peter J.; Scott, John and Wasserman, Stanley (2005) Models and Methods in Social Network Analysis, Cambridge: Cambridge University Press

Dagnino, Evelina (2003) 'Citizenship in Latin America - An Introduction', Latin American Perspectives 30: 211-25

Diani, Mario and McAdam, Doug (eds) (2005) Social Movements and Social Network Analysis, Oxford: Oxford University Press

Gerring, John (2001) Social Science Methodology: A Critical Framework, Cambridge: Cambridge University Press

Gurza Lavalle, Adrián; Houtzager, Peter P. and Acharya, Arnab (2005) 'Beyond Comparative Anecdotalism: How Civil and Political
8 Data availability in the two sectors is quite good in the cities. Measuring changes in wellbeing, however, is difficult and attribution of causality, complex. We will therefore only conduct analysis of impact on wellbeing where indicators are readily available and a consensus exists among experts of their validity. The clearest example of such an indicator is 'changes in infant mortality as an indicator for the quality of a health service'.

Organizations Shape Participation in São Paulo, Brazil', World Development 33.6: 951-61

Houtzager, Peter (2003) 'Introduction: From Policentrism to the Polity', in Peter P. Houtzager and Mick Moore (eds), Changing Paths: International Development and the New Politics of Inclusion, Ann Arbor: Michigan University Press

King, Gary; Keohane, Robert O. and Verba, Sidney (1994) Designing Social Inquiry: Scientific Inference in Qualitative Research, Princeton: Princeton University Press

Knoke, David; Urban Pappi, Franz; Broadbent, Jeffrey and Tsujinaka, Yutaka (1996) Comparing Policy Network: Labor Politics in the US, Germany, and Japan, Cambridge: Cambridge University Press

Moore, Mick; Leavy, Jennifer and White, Howard (2003) 'Polity Qualities: How Governance Affects Poverty', in Peter P. Houtzager and Mick Moore (eds), Changing Paths: International Development and the New Politics of Inclusion, Ann Arbor: Michigan University Press 